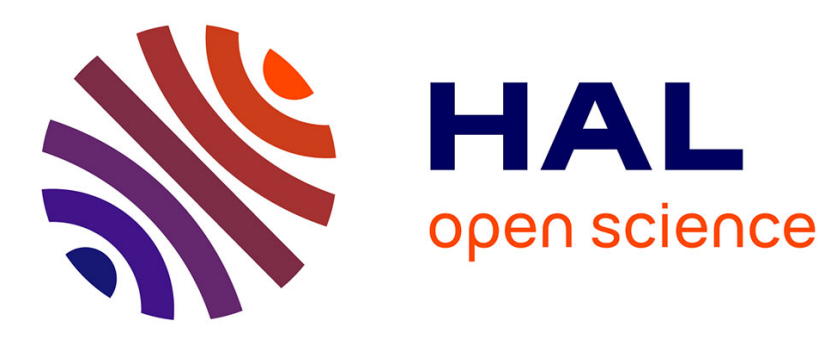

\title{
Physicochemical Characterization of Molecular Assemblies of Miltefosine and Amphotericin B
}

Cécile Menez, Philippe P. Legrand, Véronique Rosilio, Sylviane Lesieur, Gillian Barratt

\section{- To cite this version:}

Cécile Menez, Philippe P. Legrand, Véronique Rosilio, Sylviane Lesieur, Gillian Barratt. Physicochemical Characterization of Molecular Assemblies of Miltefosine and Amphotericin B. Molecular Pharmaceutics, 2007, 10.1021/mp0601143 . hal-02929439

\section{HAL Id: hal-02929439 \\ https://hal.inrae.fr/hal-02929439}

Submitted on 3 Sep 2020

HAL is a multi-disciplinary open access archive for the deposit and dissemination of scientific research documents, whether they are published or not. The documents may come from teaching and research institutions in France or abroad, or from public or private research centers.
L'archive ouverte pluridisciplinaire HAL, est destinée au dépôt et à la diffusion de documents scientifiques de niveau recherche, publiés ou non, émanant des établissements d'enseignement et de recherche français ou étrangers, des laboratoires publics ou privés. 


\title{
Physicochemical Characterization of Molecular Assemblies of Miltefosine and Amphotericin B
}

\author{
Cécile Ménez, Philippe Legrand, Véronique Rosilio, Sylviane Lesieur, and \\ Gillian Barratt* \\ Laboratoire de Physico-chimie, Pharmacotechnie et Biopharmacie, UMR CNRS 8612, \\ Univ. Paris-Sud 11, Faculté de Pharmacie, IFR 141, Châtenay-Malabry, F-92296 France
}

Received October 24, 2006; Revised Manuscript Received November 15, 2006; Accepted November 17, 2006

\begin{abstract}
This study describes the interactions between two amphiphilic molecules with antileishmanial activity, amphotericin B (AmB) and miltefosine [hexadecylphosphocholine $(\mathrm{HePC})$, the latter being effective by the oral route. The effect of HePC on the aggregation state of $A m B$ in aqueous solution and the interactions between the two agents were monitored using absorption spectroscopy and circular dichroism. Structural characterization of the mixed aggregates formed in water by dynamic light scattering (DLS) and cryofracture electron microscopy was performed. At concentrations above its critical micelle concentration, HePC was shown to interact with $A m B$, leading to an increase in the proportion of $A m B$ in its monomeric form as a result of a micellar solubilization mechanism with a capacity of $26 \pm 3 \mathrm{mmol}$ of AmB solubilized $/ \mathrm{mol}$ of $\mathrm{HePC}$, that is, nearly 40 molecules of HePC per molecule of $\mathrm{AmB}$ in the mixed micelles. These were revealed as individual and spherical aggregates close to $10 \mathrm{~nm}$ in diameter by both electron microscopy and DLS. Such a micellar formulation provides a new AmB-based system which might be useful in delivering AmB orally for visceral leishmaniasis bitherapy.
\end{abstract}

Keywords: Amphotericin B; miltefosine; hexadecylphosphocholine; micelle; solubilization

\section{Introduction}

Visceral leishmaniasis (VL), a tropical disease caused by the protozoan parasite Leishmania donovani, is usually fatal if untreated. The conventional therapy for VL with pentavalent antimonials is not very efficient due to toxic side effects and the increasing frequency of drug resistance. ${ }^{1}$ Some alternative drugs have emerged; however, to reduce and postpone the emergence of resistance, it may be appropriate to use them in combination. Among the possible candidates, amphotericin B (AmB) and miltefosine [hexadecylphosphocholine (HePC)] are particularly interesting. AmB is a very

* To whom correspondence should be addressed: Faculté de Pharmacie, Univ. Paris-Sud 11, Laboratoire de Physico-chimie, Pharmacotechnie et Biopharmacie, UMR CNRS 8612, Tour

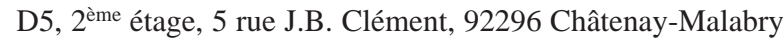
Cedex, France. E-mail : gillian.barratt@cep.u-psud.fr. Telephone: +33 (0)1 468356 27. Fax: +33 (0)1 46619334 .

(1) Ouellette, M.; Drummelsmith, J.; Papadopoulou, B. Leishmaniasis: Drugs in the Clinic. Resistance and New Developments. Drug Resist. Updates 2004, 7, 257-266. effective antileishmanial agent but has to be administered parenterally as mixed micelles in sodium deoxycholate (Fungizone), whereas HePC is the first orally active antileishmanial agent. $^{2}$

A new HePC-AmB bitherapy regimen in VL treatment would be promising for four main reasons. (i) Both drugs are amphiphilic molecules and might be expected to associate. (ii) AmB is poorly soluble in most solvents, whereas HePC, due to its lipidlike structure and its hydrophiliclipophilic balance, spontaneously forms micelles in aqueous media. ${ }^{3}$ HePC micelles could be expected therefore to solubilize AmB and create a mixed micellar system, since an important property of micelles is their ability to increase

(2) Murray, H. W. Clinical and Experimental Advances in Treatment of Visceral Leishmaniasis. Antimicrob. Agents Chemother. 2001, 45, 2185-2197.

(3) De Araujo, P. S.; Rosseneu, M. Y.; Kremer, J. M.; van Zoelen, E. J.; Haas, G. H. Structure and Thermodynamic Properties of the Complexes between Phospholipase A2 and Lipid Micelles. Biochemistry 1979, 18, 580-586. 
the aqueous solubility of hydrophobic compounds. ${ }^{4}$ Furthermore, the use of AmB is substantially limited by its severe side effects. Given that the aggregation state of $\mathrm{AmB}$ determines its toxicity toward mammalian cells, ${ }^{5}$ the solubilization of AmB aggregates by HePC micelles could lead to an attenuation of side effects, as described for various surfactants. ${ }^{6}$ (iii) We have recently demonstrated that HePC enhanced the paracellular permeability across the intestinal epithelium and could therefore improve the transport of coadministered therapeutic agents. ${ }^{7}$ Solubilization, a prerequisite for absorption by the intestinal cells, and the opening of the tight junctions induced by $\mathrm{HePC}$ molecules could improve the bioavailability of $\mathrm{AmB}$ after oral administration of a combined $\mathrm{HePC}-\mathrm{AmB}$ oral delivery system. (iv) A recent study on the interactions between miltefosine and other antileishmanial drugs has demonstrated a potentiation of HePC activity in vivo when it is used in combination with $\mathrm{AmB}$ administered by the intravenous route. ${ }^{8}$

The aim of this study was to investigate the interaction of $\mathrm{AmB}$ with the lysolecithin analogue $\mathrm{HePC}$ in an effort to propose a new delivery system for the treatment of VL. Solubilization experiments with AmB and micellar solutions of $\mathrm{HePC}$ were performed to evaluate the ability of the surfactant to include AmB in its micelles. Molecular interactions between the two compounds were probed by surface tension measurements. Structural information about the mixed water-soluble aggregates was provided by cryofracture electron microscopy and dynamic light scattering. The molecular state of AmB within the aggregates was determined by spectroscopic analysis.

\section{Materials and Methods}

2.1. Materials. Miltefosine (HePC) was from Cayman Chemical Co. (Ann Arbor, MI). Solutions of HePC (10 mM) were freshly made in water or phosphate buffer immediately before each experiment. AmB was from Bristol-Myers Squibb (Princeton, NJ). Deionized water was obtained by using a Millipore Milli-Q purification system (Millipore, Milford, MA). Analytical-grade dimethyl sulfoxide (DMSO) was from Gaylord Chemical Corp. (Bogalusa, LA), and

(4) Rangel-Yagui, C. O.; Pessoa, A., Jr.; Tavares, L. C. Micellar Solubilization of Drugs. J. Pharm. Pharm Sci. 2005, 8, 147165.

(5) Gruda, I.; Dussault, N. Effect of the Aggregation State of Amphotericin B on its Interaction with Ergosterol. Biochem. Cell Biol. 1988, 66, 177-183.

(6) Gruda, I.; Milette, D.; Brother, M.; Kobayashi, G. S.; Medoff, G.; Brajtburg, J. Structure-Activity Study of Inhibition of Amphotericin B (Fungizone) Binding to Sterols, Toxicity to Cells, and Lethality to Mice by Esters of Sucrose. Antimicrob. Agents Chemother. 1991, 35, 24-28.

(7) Ménez, C.; Buyse, M.; Chacun, H.; Farinotti, R.; Barratt, G. Modulation of Intestinal Barrier Properties by Miltefosine. Biochem. Pharmacol. 2006, 71, 486-496.

(8) Seifert, K.; Croft, S. L. In Vitro and In Vivo Interactions between Miltefosine and Other Antileishmanial Drugs. Antimicrob. Agents Chemother. 2006, 50, 73-79. methanol (MeOH) was from Carlo Erba (Val de Reuil, France). All other chemical products were from SigmaAldrich (St. Louis, MO).

2.2. Preparation of the HePC-AmB Mixtures. Micellar systems composed of $\mathrm{AmB}$ molecules incorporated into $\mathrm{HePC}$ micelles were prepared by dissolving $\mathrm{AmB}$ in $\mathrm{HePC}$ solutions. AmB at $20 \mathrm{mM}$ in DMSO was diluted in $\mathrm{MeOH}$ to $1 \mathrm{mM}$ and added to HePC solutions in PBS or water while being stirred. $\mathrm{MeOH}$ and DMSO did not exceed 5 and $0.25 \%$, respectively, of the final mixture. Finally, the binary mixtures were mixed thoroughly for $10 \mathrm{~min}$ before use. Various concentrations of AmB-loaded HePC micelles, at HePC to AmB total molar ratios between 5 and 150, were prepared.

The HePC concentration was determined enzymatically with a phospholipid detection PAP150 kit sensitive to the phosphocholine group (Biomérieux, Marcy l'Etoile, France). The AmB concentration was determined spectrophotometrically at $409 \mathrm{~nm}$ after appropriate dilution in methanol.

2.3. Absorption and Circular Dichroism Measurements. The absorption spectra of AmB were recorded using a Perkin-Elmer Lambda $11 \mathrm{UV}-$ vis spectrophotometer, while its circular dichroism (CD) spectra were recorded with a Jasco J-810 dichrograph. $\Delta \epsilon$ is the differential molar dichroic absorption coefficient $\left(\mathrm{M}^{-1} \mathrm{~cm}^{-1}\right)$. These methods have been shown to be powerful tools for studying the aggregation state of $\mathrm{AmB}$ molecules in water ${ }^{9-11}$ and interactions of $\mathrm{AmB}$ with various compounds such as lipids. ${ }^{12}$ Absorption and $\mathrm{CD}$ measurements were therefore used to monitor the influence of different concentrations of HePC on the aggregation state of $\mathrm{AmB}$ and the physicochemical interactions between the two molecules. Spectra were recorded within 30-45 min of preparation of the samples.

2.4. Solubility Measurements. The aqueous solubility of $\mathrm{AmB}$ in PBS at $\mathrm{pH} 7.4$ was determined in the presence of increasing concentrations of HePC (concentration range between 0 and $120 \mu \mathrm{M}$ ). Solubility studies were carried out using a modified Higuchi method. ${ }^{13}$ Saturated solutions were prepared by dispersing an excess amount of AmB in buffer solutions containing increasing amounts of HePC. The solutions were mixed thoroughly for $10 \mathrm{~min}$ at $23 \pm 2{ }^{\circ} \mathrm{C}$. Preliminary experiments indicated that this mixing period

(9) Bolard, J.; Seigneuret, M.; Boudet, G. Interaction between Phospholipid Bilayer Membranes and the Polyene Antibiotic Amphotericin B: Lipid State and Cholesterol Content Dependence. Biochim. Biophys. Acta 1980, 599, 280-293.

(10) Mazerski, J.; Bolard, J.; Borowski, E. Self-Association of some Polyene Macrolide Antibiotics in Aqueous Media. Biochim. Biophys. Acta 1982, 719, 11-17.

(11) Barwicz, J.; Beauregard, M.; Tancrede, P. Circular Dichroism Study of Interactions of Fungizone or AmBisome Forms of Amphotericin B with Human Low Density Lipoproteins. Biopolymers 2002, 67, 49-55.

(12) Medoff, G.; Brajtburg, J.; Kobayashi, G. S.; Bolard, J. Antifungal Agents Useful in Therapy of Systemic Fungal Infections. Annu. Rev. Pharmacol. Toxicol. 1983, 23, 303-330.

(13) Higuchi, T.; Shih, F. M.; Kimura, T.; Rytting, J. H. Solubility Determination of Barely Aqueous-Soluble Organic Solids. $J$. Pharm. Sci. 1979, 68, 1267-1272. 
was sufficient to reach equilibrium. After the incubation period, clear saturated solutions were obtained by centrifugation at $16000 \mathrm{~g}$ for $10 \mathrm{~min}$. The concentration of $\mathrm{AmB}$ was determined spectrophotometrically at $414 \mathrm{~nm}$, since this wavelength is specific for monomeric AmB bound to lipid. Phase solubility diagrams were constructed by plotting the molar concentrations of $\mathrm{AmB}$ monomers associated with $\mathrm{HePC}$ micelles as a function of the molar concentration of HePC.

2.5. Surface Tension Measurements at the Air-Water Interface. The interfacial behavior of HePC in the absence and presence of $\mathrm{AmB}$ ( 1 and $20 \mathrm{~mol} \%$ ) was studied as a function of HePC concentration. An AmB solution in DMSO $(1 \mathrm{mM})$ was diluted in triply distilled water while being stirred. The obtained aqueous solution $\left(10^{-4} \mathrm{mM}\right)$ was used to prepare all $\mathrm{HePC}-\mathrm{AmB}$ mixed solutions. The surface tension, $\gamma$, was measured by the Wilhelmy plate method using a tensiometer (K10ST, Krüss, Germany). Measurements were performed in triplicate at $23 \pm 2{ }^{\circ} \mathrm{C}$ under saturated vapor conditions. The surface tension of the water was found to be $72.3 \pm 0.2 \mathrm{mN} / \mathrm{m}$.

2.6. Structural Characterization of the $\mathrm{HePC}-\mathrm{AmB}$ Micellar System. 2.6.1. Size Measurements. The hydrodynamic diameter of $\mathrm{HePC}-\mathrm{AmB}$ mixed micelles in PBS was determined by dynamic light scattering (DLS, photon correlation spectroscopy) using a Malvern HPPS particle sizer (Malvern Instrument S.A., Orsay, France). Samples were filtered through a membrane filter with $0.45 \mu \mathrm{m}$ pores prior to processing. Measurements were taken at $25^{\circ} \mathrm{C}$ with a fixed angle of $173^{\circ}$ and diameters deduced from the StokesEinstein equation for independent spherical particles. Size distributions were derived from the autocorrelation functions using an inverse Laplace transformation. The results were expressed as the mean average over each population by assuming a log-normal distribution of the diameters.

2.6.2. Electron Microscopy. A high-pressure procedure in three steps was employed: (a) high-pressure freezing (HPF) which involves sandwich preparation and freezing of the specimen, (b) freeze-fracture (FF) which includes fracturing, replication, and cleaning of the replicas, and (c) transmission electron microscopic investigation of the replicas. For the preparation of the sandwich, a small amount of the sample was placed on a $100 \mu \mathrm{m}$ deep symmetric cup, made of copper and able to conduct heat rapidly away from the specimen, to preserve its bulk properties as long as possible.

A high-pressure cooling device (HPM 010) available from Balzers Union was used as described previously. ${ }^{14}$ The samples were subjected to a pressure of $\sim 2100$ bar from a high-pressure jet of warm liquid 2-propanol for a few milliseconds before being cooled with liquid nitrogen. After this cryofixation, the sample was maintained below its devitrification temperature in liquid nitrogen. For fracturing,

(14) Dahl, R.; Staehelin, L. A. High-Pressure Freezing for the Preservation of Biological Structure: Theory and Practice. $J$. Electron Microsc. Tech. 1989, 13, 165-174. the sandwiches were mounted on a cold table, which was inserted inside the vacuum chamber of a Balzers model BAF 400T apparatus on a nitrogen-cooled support kept at $103 \mathrm{~K}$. Once the vacuum was lower than $10^{-7}$ Torr, fracturing was achieved by displacing a single-edge scalpel blade precooled to $83 \mathrm{~K}$.

The replication of the surface was performed in three steps. First, a thin layer $(2 \mathrm{~nm})$ of platinum was evaporated onto the specimen from a shadowing angle of $45^{\circ}$ to provide contrast enhancement of the topographic features of the fracture surface. The second step consisted of a rotary shadowing of similar thickness. This allowed portions of the specimen that might otherwise have been obscured by the shadow to be resolved. Finally, a 20 nm layer of carbon was deposited to strengthen the shadow cast. All film thicknesses were monitored by a quartz crystal thickness gauge (4.96 MHz, quartz crystal holder QSK 301 and monitor QSG 060, Baltec). After complete deposition, the vacuum chamber was vented and the specimens were removed. The replicas of the surfaces were then floated off the specimens by submerging them in a $70 \%$ sulfuric acid solution and collected onto naked 400 mesh grids, which were subsequently mounted in a transmission electron microscope for inspection. Transmission electron microscopy (TEM) measurements were performed on a LEO 912 Omega high-resolution microscope working at $120 \mathrm{kV}$.

2.7. Statistics. All experiments were conducted at least in triplicate, and results are expressed as means \pm the standard deviation (SD). Statistical analysis was performed using one-way analysis of variance (ANOVA) with a MannWhitney post-test for double comparisons or a Student's $t$ test (GraphPad Instat, San Diego, CA). Statistical significance was accepted as $p<0.05$.

\section{Results}

3.1. Spectral Analysis. 3.1.1. Effect of HePC above Its Critical Micelle Concentration (CMC) on the AmB Aggregation State. The UV-visible spectroscopy technique is particularly useful for characterizing AmB formulations since it has been shown to be very sensitive to the aggregation state of $\mathrm{AmB}$ and to interactions of the drug with other molecules. ${ }^{15}$ Figure $1 \mathrm{~A}$ presents the UV-visible spectra for $\mathrm{AmB}$ at a constant concentration of $10 \mu \mathrm{M}$ (i.e., above its $\mathrm{CMC}$ ) alone in solution or mixed with $\mathrm{HePC}$ at various $\mathrm{HePC} / \mathrm{AmB}$ molar ratios $(5,20$, and 50, i.e., above the $\mathrm{CMC}$ of $\mathrm{HePC}$ reported to be $\sim 10 \mu \mathrm{M}){ }^{3,16-18}$ The spectrum of $\mathrm{AmB}$ alone in water exhibited peaks typical of

(15) Barwicz, J.; Gruszecki, W. I.; Gruda, I. Spontaneous Organization of Amphotericin B in Aqueous Medium. J. Colloid Interface Sci. 1993, 158, 71-76.

(16) Unger, C.; Eibl, H. Hexadecylphosphocholine: Preclinical and the First Clinical Results of a New Antitumor Drug. Lipids 1991, 26, 1412-1417.

(17) Matos, C.; Chaimovich, H.; Lima, J. L.; Cuccovia, I. M.; Reis, S. Effect of Liposomes on the Rate of Alkaline Hydrolysis of Indomethacin and Acemetacin. J. Pharm. Sci. 2001, 90, 298309. 

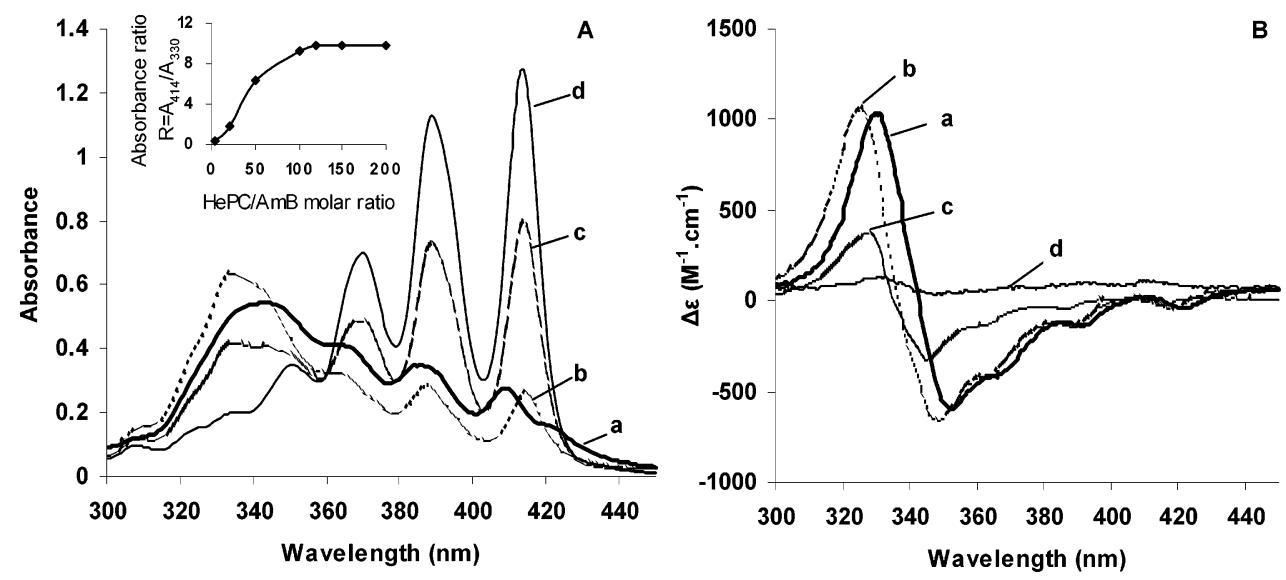

Figure 1. Absorption $(\mathrm{A})$ and circular dichroic $(\mathrm{B})$ spectra of $\mathrm{AmB}$ (total concentration of $10 \mu \mathrm{M})$ in aqueous solution (a) and in aqueous mixtures with $\mathrm{HePC}$ at $\mathrm{HePC} / \mathrm{AmB}$ molar ratios of 5 (b), 20 (c), and 50 (d). The spectra were recorded 45 min after preparation of solutions and are representative of three experiments. The inset shows the $A_{414} / A_{330}$ absorbance ratio as a function of HePC content.

AmB in both aggregated and monomeric forms: an absorption band at $\sim 409 \mathrm{~nm}$, characteristic of the monomeric form in water, and an absorption band at $\sim 328 \mathrm{~nm}$, characteristic of the aggregated state. ${ }^{19-21}$ When increasing concentrations of HePC were added, several changes in the absorption spectrum were observed, as shown in Figure 1A. The presence of $\mathrm{HePC}$ at a $\mathrm{HePC} / \mathrm{AmB}$ molar ratio of 5 induced (i) a small increase in the band intensity at $328 \mathrm{~nm}$ compared with the spectrum of $\mathrm{AmB}$ alone, suggesting that the AmB aggregates had been reorganized, and (ii) a shift of the intensity maximum from 409 to $414 \mathrm{~nm}$. This demonstrates that the environment of the AmB molecule in its monomeric form was significantly changed and therefore suggests its insertion into the HePC micelles. Further addition of HePC led to (iii) a decrease in the magnitude of the absorption band at $330 \mathrm{~nm}$ with a concomitant increase in the magnitude of the absorption band at $414 \mathrm{~nm}$, indicating a decrease in the level of self-aggregated AmB and an increase in the level of monomeric $\mathrm{AmB}$ bound to $\mathrm{HePC}$. The proportion of monomeric $\mathrm{AmB}$ may be obtained from the $A_{414} / A_{330}$ ratio of absorbance. ${ }^{22,23}$ As shown in the inset, this ratio increased with an increase in the $\mathrm{HePC} / \mathrm{AmB}$ molar ratio, demonstrating that the $\mathrm{AmB}$ aggregates were destroyed and that $\mathrm{AmB}$ molecules became more and more individualized so that the

(18) Yaseen, M.; Wang, Y.; Su, T. J.; Lu, J. R. Surface Adsorption of Zwitterionic Surfactants: n-Alkyl Phosphocholines Characterised by Surface Tensiometry and Neutron Reflection. J. Colloid Interface Sci. 2005, 288, 361-370.

(19) Kawabata, M.; Onda, M.; Mita, T. Effect of Aggregation of Amphotericin B on Lysophosphatidylcholine Micelles as Related to its Complex Formation with Cholesterol or Ergosterol. $J$. Biochem. 2001, 129, 725-732.

(20) Milhaud, J.; Michels, B. Binding of Nystatin and Amphotericin B with Sterol-Free L-Dilauroylphosphatidylcholine Bilayers Resulting in the Formation of Dichroic Lipid Superstructures. Chem. Phys. Lipids 1999, 101, 223-235.

(21) Barwicz, J.; Dumont, I.; Ouellet, C.; Gruda, I. Amphotericin B Toxicity as Related to the Formation of Oxidatively Modified Low-Density Lipoproteins. Biospectroscopy 1998, 4, 135-144. absorbance ratio reached a maximum plateau value close to 10 when the $\mathrm{HePC} / \mathrm{AmB}$ molar ratio was $\geq 120$. At this stage, AmB was predominantly dissociated into its monomeric form and presumably dissolved by the surfactant micelles.

Figure 1B presents the CD spectra of the same preparations. These results were in agreement with those of UVvisible measurements: the addition of HePC to a solution of $\mathrm{AmB}$ in water induced various changes in the $\mathrm{CD}$ spectrum, including (i) a shift of the intense dichroic doublet centered around $340-334 \mathrm{~nm}$, demonstrating an interaction between $\mathrm{AmB}$ and $\mathrm{HePC}$ at a molar ratio of 5, and (ii) the decrease of this excitonic doublet, characteristic of selfassociated $\mathrm{AmB},{ }^{24,25}$ with a further increase in HePC concentration, clearly demonstrating the reduction of the proportion of aggregates. ${ }^{26}$ When the HePC concentration was increased further $(\mathrm{HePC} / \mathrm{AmB}$ molar ratio of $>120)$, the dichroic doublet disappeared completely (data not shown). Taken together, these results clearly demonstrate that HePC can interact with $\mathrm{AmB}$ monomers and thus disperse $\mathrm{AmB}$ aggregates.

3.1.2. Effect of HePC below Its CMC on the AmB Aggregation State. When the concentration of HePC added was below its CMC $(10-12 \mu \mathrm{M}$ as indicated in the

(22) Gruda, I.; Gauthier, E.; Elberg, S.; Brajtburg, J.; Medoff, G. Effects of the Detergent Sucrose Monolaurate on Binding of Amphotericin B to Sterols and its Toxicity for Cells. Biochem. Biophys. Res. Commun. 1988, 154, 954-958.

(23) Brajtburg, J.; Gruda, I.; Daigle, I.; Medoff, G. Concentration Dependant Dual Effect of the Monolauryl Ester of Sucrose on the Antifungal Activity and Absorption Spectra of Amphotericin B (Fungizone). Biochim. Biophys. Acta 1989, 985, 307-312.

(24) Brittain, H. G. Circular Dichroism Studies of the Self-Association of Amphotericin B. Chirality 1994, 6, 665-669.

(25) Romanini, D.; Avalle, G.; Nerli, B.; Pico, G. Thermodynamic and Spectroscopic Features of the Behavior of Amphotericin B in Aqueous Medium. Biophys. Chem. 1999, 77, 69-77.

(26) Bolard, J.; Cheron, M. Association of the Polyene Antibiotic Amphotericin B with Phospholipid Vesicles: Perturbation by Temperature Changes. Can. J. Biochem. 1982, 60, 782-789. 


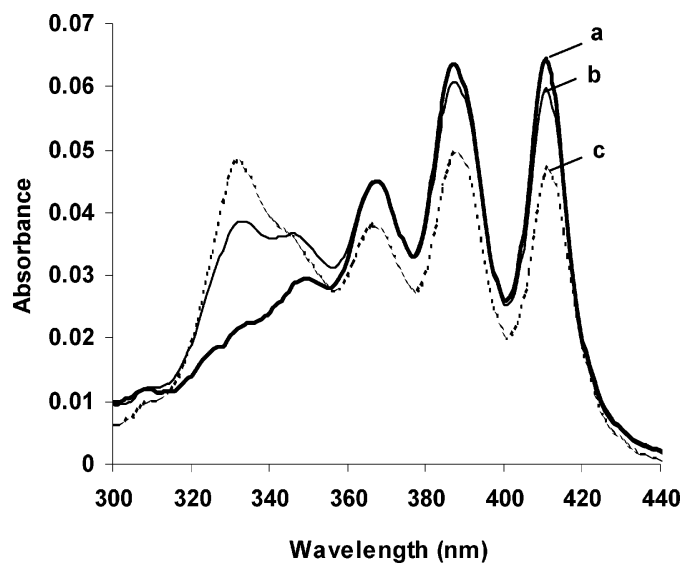

Figure 2. Effect of $\mathrm{HePC}$ at concentrations below its $\mathrm{CMC}$ on the absorption spectrum of AmB (total concentration of 1 $\mu \mathrm{M})$ : (a) AmB alone in water, (b) $\mathrm{HePC} / \mathrm{AmB}$ molar ratio of 4 , and (c) HePC/AmB molar ratio of 8 . The spectra were recorded 45 min after mixing and are representative of three experiments.

literature), ${ }^{3,16,17}$ the modifications of $\mathrm{AmB}$ spectra were different from those observed above the $\mathrm{CMC}$, suggesting that the molecular environment around the AmB molecules was different in the two cases. Figure 2 showed that when $\mathrm{HePC}$ was added to a $1 \mu \mathrm{M} \mathrm{AmB}$ solution (above the CMC of the antibiotic, $\sim 0.1 \mu \mathrm{M})^{27}$ at molar ratios of 4 and 8 , so that the HePC concentration was below $10 \mu \mathrm{M}$, the band at $330 \mathrm{~nm}$ increased in amplitude while the band characteristic of the monomeric form of $\mathrm{AmB}$ decreased in intensity. From the comparison with spectrum in Figure $1 \mathrm{~A}$ recorded at 10 $\mu \mathrm{M} \mathrm{AmB}$, the addition of $\mathrm{HePC}$ monomers to $1 \mu \mathrm{M} \mathrm{AmB}$ seems to favor the aggregation of AmB. Furthermore, the band at $409 \mathrm{~nm}$ was not shifted toward $414 \mathrm{~nm}$, demonstrating that no interaction between $\mathrm{AmB}$ monomers and $\mathrm{HePC}$ molecules occurred. These findings strongly suggest that the solubility of AmB monomers in water was reduced in the presence of HePC monomers which competed for hydration by water molecules. This is supported by previous studies which have shown that the water solubility of different surfactants as a mixture is lower than that of each of them separately. ${ }^{28}$

3.2. Micellar Solubilization Mechanism and Solubility Measurements. To further characterize the interaction of $\mathrm{AmB}$ with $\mathrm{HePC}$, and to determine whether these spectral changes reflected the formation of mixed aggregates of defined composition, the solubility of AmB was measured precisely. The measurements were made with $\mathrm{AmB}$ in excess, leading to a three-phase system: nondissolved AmB, $\mathrm{AmB}$, and $\mathrm{HePC}$ in solution and mixed micelles. Figure 3 shows the phase solubility diagram, constructed by plotting the molar concentration of $\mathrm{AmB}$ solubilized, i.e., bound to

(27) Barwicz, J.; Christian, S.; Gruda, I. Effects of the Aggregation State of Amphotericin B on its Toxicity to Mice. Antimicrob. Agents Chemother. 1992, 36, 2310-2315.

(28) Clint, J. H. Micellization of Mixed Nonionic Surface Active Agents. J. Chem. Soc., Faraday Trans. 1975, 71, 1327-1334.

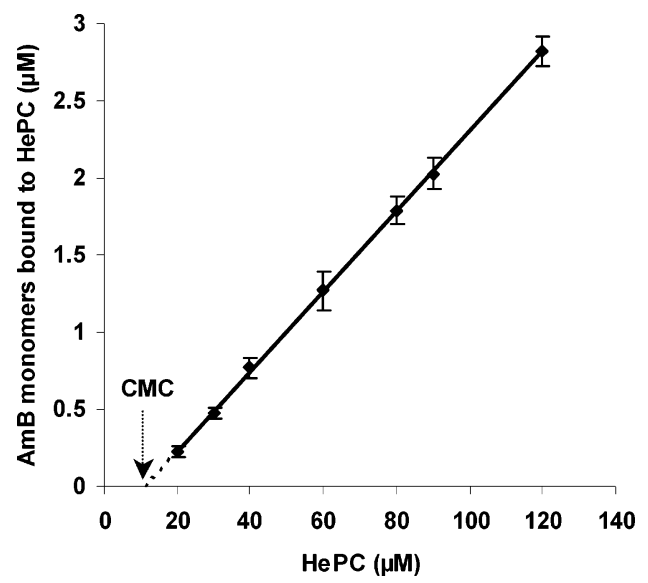

Figure 3. Solubility profile of $\mathrm{AmB}$ in the presence of increasing concentrations of HePC. The slope of the solubility line was $0.0259 \pm 0.0031\left(R^{2}=0.999\right)$, corresponding to an $\mathrm{AmB}$ monomers/HePC ratio in the mixed aggregates close to $1 / 40$. All measurements were expressed as means $\pm S D$ of three determinations with three different preparations $(n=$ 9).

$\mathrm{HePC}$ in its monomeric form, versus the molar concentration of $\mathrm{HePC}$, after the former was added to solutions of the latter at various concentrations. The solubility of AmB increased in a linear manner $\left(R^{2}=0.999\right)$ with HePC concentration, typical of micellar solubilization. ${ }^{4}$ The stoichiometry of the $\mathrm{AmB}-\mathrm{HePC}$ mixed micelles was determined from these Higuchi plots as the slope of the solubility line ${ }^{29}$ and was found to be close to one molecule of $\mathrm{AmB}$ associated with $40 \mathrm{HePC}$ molecules. The threshold concentration of HePC for $\mathrm{AmB}$ association was determined by extrapolation to be $11.1 \pm 0.1 \mu \mathrm{M}$.

3.3. Surface Tension Measurements. Surface tension measurements were performed to determine the CMC of $\mathrm{HePC}$ and to analyze its interaction with $\mathrm{AmB}$ at various concentrations. The surface tension, $\gamma$, of HePC solutions in the absence or presence of $\mathrm{AmB}(\mathrm{HePC} / \mathrm{AmB}$ molar ratios of 5 and 100) was measured for HePC concentrations in the range of $10^{-8}-10^{-3} \mathrm{M}$. The results are plotted in Figure 4. As expected for a soluble surfactant, the addition of HePC to the aqueous phase resulted in a concentration-dependent decrease in the surface tension value until it reached 38.5 $\mathrm{mN} / \mathrm{m}$ and remained constant afterward. The CMC of HePC, corresponding to the concentration at which the air-water interface was saturated by surfactant molecules, was found to be $11 \pm 2 \mu \mathrm{M}$, in agreement with the values previously reported in the literature. ${ }^{3,18}$

When $\mathrm{AmB}$ was added to aqueous solutions of $\mathrm{HePC}$ at the $\mathrm{HePC} / \mathrm{AmB}$ molar ratios of 5 and 100 , the surface tension of the mixtures (for each HePC concentration that was studied) appeared almost identical to that of HePC alone

(29) Singh, P.; Desai, S. J.; Flanagan, D. R.; Simonelli, A. P.; Higuchi, W. I. Mechanistic Study of the Influence of Micelle Solubilization and Hydrodynamic Factors on the Dissolution Rate of Solid Drugs. J. Pharm. Sci. 1968, 57, 959-965. 


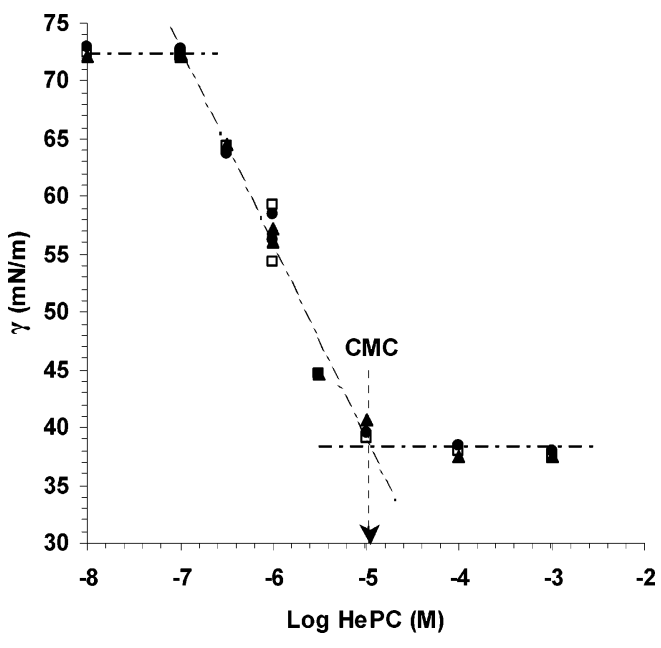

Figure 4. Interfacial behavior of $\mathrm{HePC}$ and $\mathrm{HePC}-\mathrm{AmB}$ mixtures. Surface tension $(\gamma)$ was measured as a function of HePC concentration (molar), alone $(\bullet)$ or in a mixture with $\mathrm{AmB}$ at $\mathrm{HePC} / \mathrm{AmB}$ molar ratios of 100 ( $\square$ ) or 5 ( $\mathbf{\Delta})$. Measurements were performed in triplicate at $23 \pm 2{ }^{\circ} \mathrm{C}$.

(Figure 4). Clearly, the adsorption of HePC monomers was not significantly affected by the presence of AmB: the two compounds did not form a mixed monolayer at the airsolution interface, and the $\mathrm{CMC}$ of $\mathrm{HePC}$ remained unmodified.

3.4. Characterization of HePC-AmB Micellar Systems. 3.4.1. Size Measurements. The average hydrodynamic diameter of HePC micelles in solution was measured by dynamic light scattering and found to be $7 \pm 0.2 \mathrm{~nm}$. This size was independent of the $\mathrm{HePC}$ concentration in the range of $20 \mu \mathrm{M}$ to $20 \mathrm{mM}$ when above the CMC. A mean hydrodynamic diameter of $80 \pm 5 \mathrm{~nm}$ was measured on a $10 \mu \mathrm{M}$ aqueous solution of AmB. The average size of the putative $\mathrm{HePC}-\mathrm{AmB}$ micellar systems was then investigated in various combinations with a constant concentration of $\mathrm{AmB}$ of $10 \mu \mathrm{M}$ and increasing concentrations of HePC (HePC/AmB molar ratio range from 5 to 200). The results are shown in Figure 5. In the HePC-AmB mixtures, two populations of aggregates were observed: the first one with a mean hydrodynamic diameter of $210 \pm 30 \mathrm{~nm}$ and the second one around $7 \mathrm{~nm}$. The analysis of the scattering intensity from each of these populations showed a decrease in the proportion of the larger aggregates and a corresponding increase in the proportion of the smaller aggregates with an increasing $\mathrm{HePC}$ concentration.

3.4.2. Morphology by Cryofracture Electron Microscopy. Cryofracture electron microscopy is a good method for visualizing nanosized objects and was therefore used to obtain information complementary to the light scattering experiments. Figure 6 shows representative electron micrographs of $\mathrm{HePC}$ and $\mathrm{HePC}-\mathrm{AmB}$ mixture solutions at $\mathrm{HePC} / \mathrm{AmB}$ molar ratios of 5 or 100 . As expected, alone in solution, HePC was in the form of very small spherical or spheroid micelles (Figure 6A). The isolated micelles observed under these conditions had a mean diameter of $10 \mathrm{~nm}$. When $\mathrm{AmB}$ was present in the solution at a $\mathrm{HePC} / \mathrm{AmB}$ molar ratio

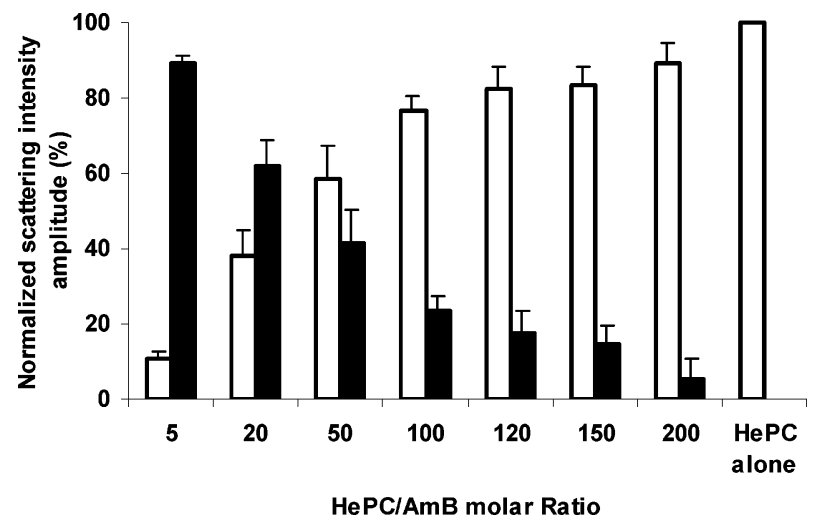

Figure 5. Normalized light scattering intensity amplitude as a function of $\mathrm{HePC} / \mathrm{AmB}$ molar ratio. The $\mathrm{AmB}$ concentration was fixed at $10 \mu \mathrm{M}$, and the HePC concentration was increased from $50 \mu \mathrm{M}$ to $2 \mathrm{mM}$. Two different populations were considered: mean diameter of $7 \mathrm{~nm}$ (white bars) and mean diameter of $210 \mathrm{~nm}$ (black bars). All measurements were expressed as means \pm SD of three determinations with three different samples $(n=9)$.

of 5 (Figure 6B), numerous larger aggregates (black arrowheads) of various sizes $(50-100 \mathrm{~nm})$ were revealed, coexisting with smaller spherical micelle-like aggregates (white arrows). When the $\mathrm{HePC} / \mathrm{AmB}$ molar ratio was increased to 100 (Figure 6C), some large aggregates were still observed, but their mean size and proportion were significantly reduced while the small micelle-like aggregates (diameter of $\sim 10 \mathrm{~nm}$ ) persisted.

\section{Discussion}

The aim of this study was to conceive and characterize a mixed micellar system composed of $\mathrm{HePC}$ and $\mathrm{AmB}$ for bitherapy in visceral leishmaniasis treatment. We therefore investigated the physicochemical interactions between these two antileishmanial agents.

The degree of aggregation of $\mathrm{AmB}$ and its interaction with $\mathrm{HePC}$ were monitored by its absorption and circular dichroism spectra, as had already been done for its interactions with other lipids. ${ }^{26,27}$ Our results indicate that HePC interacts with $\mathrm{AmB}$, as shown by the shift of both the absorption maximum and the dichroic doublet. A similar result has already been observed with surfactants like Myrj 59, a poly(ethylene oxide) derivative of stearic acid, ${ }^{30}$ or sodium deoxycholate ${ }^{31}$ and has been interpreted as the binding of $\mathrm{AmB}$ in its monomeric form to the amphiphilic lipid, when above its CMC. Furthermore, $\mathrm{HePC}$ is a structural analogue of phospholipids, and the shift of the AmB monomeric absorbance observed in our study from 409 to $414 \mathrm{~nm}$ has also been found for $\mathrm{AmB}-$ phospholipid mixed systems and

(30) Tasset, C.; Preat, V.; Roland, M. The Influence of Myrj 59 on the Solubility, Toxicity and Activity of Amphotericin B. J. Pharm. Pharmacol. 1991, 43, 297-302.

(31) Tancrede, P.; Barwicz, J.; Jutras, S.; Gruda, I. The effect of Surfactants on the Aggregation State of Amphotericin B. Biochim. Biophys. Acta 1990, 1030, 289-295. 


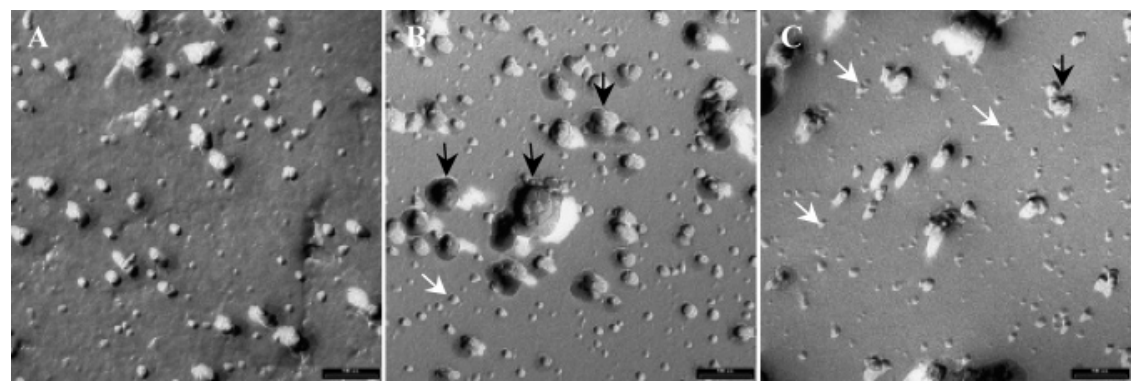

Figure 6. Cryofracture electron microscopy of $\mathrm{HePC}$ and $\mathrm{HePC}-\mathrm{AmB}$ mixtures. (A) HePC solution $(20 \mathrm{mM})$ in water showing only very small spherical micelles. (B) HePC/AmB mixtures at a molar ratio of $5(0.2 \mathrm{mM} \mathrm{AmB}$ and $1 \mathrm{mM} \mathrm{HePC})$ showing large aggregates (black arrows) coexisting with small spherical micelles (white arrows). (C) HePC/AmB mixtures at a molar ratio of $100(0.2 \mathrm{mM} \mathrm{AmB}$ and $20 \mathrm{mM} \mathrm{HePC}$ ) showing aggregates (black arrows) coexisting with small spherical micelles (white arrows) in higher proportion. The scale bar is $100 \mathrm{~nm}$.

shown to be characteristic of a specific interaction between AmB monomers and phospholipid molecules. ${ }^{32}$ Thus, this shift confirms that $\mathrm{HePC}$ in its micellar form interacts strongly with $\mathrm{AmB}$ monomers. HePC above its $\mathrm{CMC}$ was also found to induce a change in the molecular organization of AmB from being mainly in its aggregated form to a higher proportion of it being in the monomeric form. These modifications of the AmB spectrum were similar to those induced by ethano ${ }^{33}$ and various solubilizing surfactants with totally different chemical structures, including monolauryl ester of sucrose and palmitoyl mannose, ${ }^{22,23}$ Myrj 59, ${ }^{30}$ Tween 80 , and sodium deoxycholate, ${ }^{31}$ and are known to be due to a decrease in the degree of aggregation of the AmB molecules. ${ }^{27}$ Thus, HePC, an antileishmanial agent, is able to modify the aggregation state of $\mathrm{AmB}$ in solution in such a manner that it solubilizes AmB within its micelles. This result is biologically relevant since experimental evidence has been accumulated over the past decade which shows a close correlation between the aggregation state of $\mathrm{AmB}$ in the delivery system and its toxicity. ${ }^{22,31,34}$ Indeed, it has been proposed that monomeric $\mathrm{AmB}$ is nontoxic to mammalian cells but causes fungal cell death by membrane damage, presumably due to its selective interaction with ergosterol, while aggregated $\mathrm{AmB}$ is nonselective, forming pores in both mammalian and fungal or parasite cell membranes. ${ }^{34}$ The micellar system composed of $\mathrm{HePC}$ and $\mathrm{AmB}$ could therefore reduce $\mathrm{AmB}$ toxicity toward mammalian cells.

The interactions between $\mathrm{HePC}$ and $\mathrm{AmB}$ observed by spectral analysis can be summarized as follows. At low HePC concentrations (below and around its critical micelle concentration), the HePC molecules free in solution do not improve the solubility of $\mathrm{AmB}$, as judged by its absorption spectrum. At higher concentrations of $\mathrm{HePC}$ above the CMC,

(32) Fournier, I.; Barwicz, J.; Tancrede, P. The Structuring Effects of Amphotericin B on Pure and Ergosterol- or Cholesterol-Containing Dipalmitoylphosphatidylcholine Bilayers: A Differential Scanning Calorimetry Study. Biochim. Biophys. Acta 1998, 1373, 76-86.

(33) Rinnert, H.; Thirion, C.; Dupont, G.; Lematre, J. Structural Studies on Aqueous and Hydroalcoholic Solutions of a Polyene Antibiotic: Amphotericin B. Biopolymers 1977, 16, 2419-2427.

(34) Brajtburg, J.; Bolard, J. Carrier Effects on Biological Activity of Amphotericin B. Clin. Microbiol. Rev. 1996, 9, 512-531. the AmB aggregates are gradually destroyed. A further increase in HePC concentration leads to the formation of mixed micelles composed of HePC molecules with incorporated monomeric $\mathrm{AmB}$. At high concentrations, the mixed aggregates coexist with pure $\mathrm{HePC}$ micelles that serve as a pool of $\mathrm{HePC}$ and stabilize the mixed aggregate. Dilution below the CMC of HePC leads to a destabilization of the aggregates (data not shown). Monitoring the $A_{414} / A_{330}$ absorbance ratio demonstrated that a complete dissolution of $\mathrm{AmB}$ aggregates was reached with a $\mathrm{HePC} / \mathrm{AmB}$ molar ratio of 120, at the concentrations that were studied. Lysophosphatidylcholine (LPC) was previously found to act in the same way: in LPC micelles, $90 \%$ or more of the AmB was in a monomeric form when the $\mathrm{LPC} / \mathrm{AmB}$ ratio approached $200 .{ }^{19}$

The phenomenon of micellar solubilization of $\mathrm{AmB}$ aggregates by $\mathrm{HePC}$ micelles was confirmed by size measurements and cryofracture electron microscopy observation of $\mathrm{HePC}-\mathrm{AmB}$ mixtures. Indeed, $\mathrm{AmB}$ in aqueous solution was found to form rather large aggregates whose mean size was $\sim 80 \mathrm{~nm}$, in agreement with previous findings describing them as giant micelles ${ }^{35}$ or precipitated aggregates of $\sim 2000$ molecules of AmB. ${ }^{33}$ The addition of HePC above its $\mathrm{CMC}$ to $\mathrm{AmB}$ aqueous dispersions did not lead to a reduction in the size of the $\mathrm{AmB}$ aggregates but did decrease their proportion in the preparation. When more HePC was added, small micelles were the predominant objects present in the $\mathrm{HePC}-\mathrm{AmB}$ mixture. These micelles, the diameter of which was $\sim 7 \mathrm{~nm}$, probably represent a mixture of pure $\mathrm{HePC}$ micelles and $\mathrm{HePC}-\mathrm{AmB}$ mixed micelles. These observations strongly suggest that $\mathrm{AmB}$ molecules do not modify the Stokes radius for the hydrated HePC micelles. Indeed, the diameter for the pure $\mathrm{HePC}$ micelle of $7.0 \pm$ $0.2 \mathrm{~nm}$ measured in our study is in accordance with the Stokes radius for the hydrated micelles that was found to be $3.4 \pm 0.2 \mathrm{~nm} .{ }^{3}$ This was confirmed by electron microscopy. The apparent overestimate of the micelle size of $\sim 3 \mathrm{~nm}$

(35) Egito, E. S.; Araujo, I. B.; Damasceno, B. P.; Price, J. C. Amphotericin B/Emulsion Admixture Interactions: An Approach Concerning the Reduction of Amphotericin B Toxicity. J. Pharm. Sci. 2002, 91, 2354-2366. 
compared to that of DLS was probably due to the thin layer of platinum added during the replica formation. Moreover, it should be noted that the addition of HePC to AmB, even at the low molar ratio of 5 , induced the formation of a new type of aggregate, changing in size from $80 \mathrm{~nm}$ in the case of pure AmB aggregates to $210 \mathrm{~nm}$ in the case of $\mathrm{HePC}-$ AmB mixtures.

The micellar solubilization mechanism was then characterized. We demonstrated that the molar solubilization capacity was close to $26 \mathrm{mmol}$ of $\mathrm{AmB}$ solubilized by $1 \mathrm{~mol}$ of $\mathrm{HePC}$ (i.e., a micellar solubilization ratio of $2.6 \%$ ), leading to a micellar system composed of one $\mathrm{AmB}$ molecule for approximately $40 \mathrm{HePC}$ molecules. This composition was also achieved when excess HePC was added (data not shown), giving further evidence of a solubilization of $\mathrm{AmB}$ in monomeric form in micelles of defined structure. Since it has been demonstrated previously that HePC micelles have an aggregation number of 155 monomers, ${ }^{3}$ it can be assumed that approximately three or four $\mathrm{AmB}$ molecules are associated with one $\mathrm{HePC}$ micelle. The threshold concentration of $\mathrm{HePC}$ for $\mathrm{AmB}$ association of $\sim 11.1 \mu \mathrm{M}$ determined from solubility measurements is comparable with the CMC estimated from surface tension measurements $(11 \pm 2 \mu \mathrm{M})$ and confirms the hypothesis of micellar solubilization. The solubility of AmB is increased by $\mathrm{HePC}$ at or above its CMC, indicating that $\mathrm{AmB}$ monomers associate with $\mathrm{HePC}$ only in its micellar form.

In this study, we showed that HePC behaved like a number of other surfactants in its capacity to solubilize AmB. However, the presence of $\mathrm{AmB}$ in solution did not affect the interfacial behavior of $\mathrm{HePC}$, at the concentrations that were studied. It has been demonstrated that the influence of $\mathrm{AmB}$ on the surfactant $\mathrm{CMC}$ depends on the nature of the interactions between $\mathrm{AmB}$ and the surfactant molecules. ${ }^{31}$ Our result could therefore indicate that the interaction between $\mathrm{HePC}$ and $\mathrm{AmB}$ was not sufficient to perturb the formation of HePC micelles, which leads to a hypothesis about the localization of $\mathrm{AmB}$ within the micelles. Indeed, no competition was found between $\mathrm{HePC}$ and $\mathrm{AmB}$ at the air-water interface, despite the surface properties of both compounds, suggesting that no insertion of the AmB molecule in the HePC monolayer occurred. Moreover, it is unlikely that $\mathrm{AmB}$ would be dissolved in the hydrophobic core of the micelle, given its poor solubility in apolar solvents. The shift in the absorbance maximum characteristic of the monomeric form of $\mathrm{AmB}$ from 409 to $414 \mathrm{~nm}$ was almost identical to that observed when AmB was associated with phospholipid assemblies. Thus, it is reasonable to conclude that $\mathrm{AmB}$ monomers associate with the $\mathrm{HePC}$ micelles, close to the polar head group of the surface-active compound.

The limiting area per molecule $(A)$ for $\mathrm{HePC}$ was calculated using the simplified Gibbs equation $\Gamma_{i}=(-1 /$ $R T)\left\{\mathrm{d} \gamma / \mathrm{d}\left[\ln \left(C_{i}\right)\right]\right\}$, where $\Gamma$ is the interfacial concentration, and the relationship between $A$ and $\Gamma, A=1 / N_{\mathrm{a}} \Gamma$, where $N_{\mathrm{a}}$ is Avogadro's number. The molecular area of HePC was found to be $41.8 \AA^{2}$, which is much larger than that of a fatty acid $\left(25 \AA^{2}\right)$. However, the limiting molecular area, deduced from HePC compression isotherms, was previously found to be $26 \AA^{2}{ }^{26}$ demonstrating that in our study, the $\mathrm{HePC}$ monolayers formed under equilibrium conditions at the air-solution interface were much more expanded than those formed under dynamic conditions of compression. This could be due to the organization of the molecules in the uncompressed monolayers with their hydrocarbon tails tilted with respect to the plane of the interface, ${ }^{37}$ which could hinder the penetration of $\mathrm{AmB}$ at the interface. Similar behavior was reported by Minones et al., who compared adsorption of AmB into DPPC and DOPC monolayers. They showed that $\mathrm{AmB}$ adsorbed to a much lesser extent into the expanded DOPC monolayers than into the densely packed DPPC ones. ${ }^{38,39}$ They also showed that apart from the packing of a spread monolayer, the intermolecular interactions of a lipid with AmB should be taken into account, such as those between the zwitterionic polar headgroup of a phospholipid and the carboxyl and ammonium groups of AmB.

In summary, we have demonstrated in this study that $\mathrm{HePC}$, when present above its $\mathrm{CMC}$, (i) can solubilize $\mathrm{AmB}$ aggregates and (ii) allows formation of $\mathrm{HePC}-\mathrm{AmB}$ mixed micellar systems, probably composed of three to four $\mathrm{AmB}$ molecules adsorbed at the surface of HePC micelles. These results may have important clinical implications for the conception of a new oral delivery system for bitherapy of leishmaniasis. The biological effects of this $\mathrm{HePC}-\mathrm{AmB}$ association are currently under investigation in vitro.

Acknowledgment. We gratefully acknowledge MarieMartine Boissonnade for surface tension measurements and Monique Chéron for circular dichroism measurements. We also thank the service of Electron Microscopy of IFR 83 "Biologie Integrative" and the Ile-de-France region for access to the high-pressure machine and the TEM LEO 912 Omega. We are indebted to G. Frebourg and J. P. Lechaire for the excellent preparation of the replicas.

MP0601143

(36) Rakotomanga, M.; Loiseau, P. M.; Saint-Pierre-Chazalet, M. Hexadecylphosphocholine Interaction with Lipid Monolayers. Biochim. Biophys. Acta 2004, 1661, 212-218.

(37) Rey Gomez-Serranillos, I.; Minones, J., Jr.; Dynarowicz-Latka, P.; Iribarnegaray, E.; Casas, M. Study of the p-A Isotherms of Miltefosine Monolayers Spread at the Air/Water Interface. Phys. Chem. Chem. Phys. 2004, 6, 1580-1586.

(38) Minones, J., Jr.; Conde, O.; Dynarowicz-Latka, P.; Casas, M. Penetration of Amphotericin B into DOPC Monolayers Containing Sterols of Cellular Membranes. Colloids Surf., A 2005, 270-271, 129-137.

(39) Dynarowicz-Latka, P.; Seoane, R.; Minones, J., Jr.; Velo, M.; Minones, J. Study of Penetration of Amphotericin B into Cholesterol or Ergosterol Containing Dipalmitoyl Phosphatidylcholine Langmuir Monolayers. Colloids Surf., B 2002, 27, 249263 